This item was submitted to Loughborough's Research Repository by the author.

Items in Figshare are protected by copyright, with all rights reserved, unless otherwise indicated.

\title{
Computational modelling of the anisotropic conductive adhesive assembly process
}

PLEASE CITE THE PUBLISHED VERSION

PUBLISHER

(c) IEEE

LICENCE

CC BY-NC-ND 4.0

REPOSITORY RECORD

Whalley, David C., Greg Glinsky, Chris Bailey, and Johan Liu. 2019. "Computational Modelling of the Anisotropic Conductive Adhesive Assembly Process". figshare. https://hdl.handle.net/2134/3980. 
This item was submitted to Loughborough's Institutional Repository (https://dspace.lboro.ac.uk/) by the author and is made available under the following Creative Commons Licence conditions.

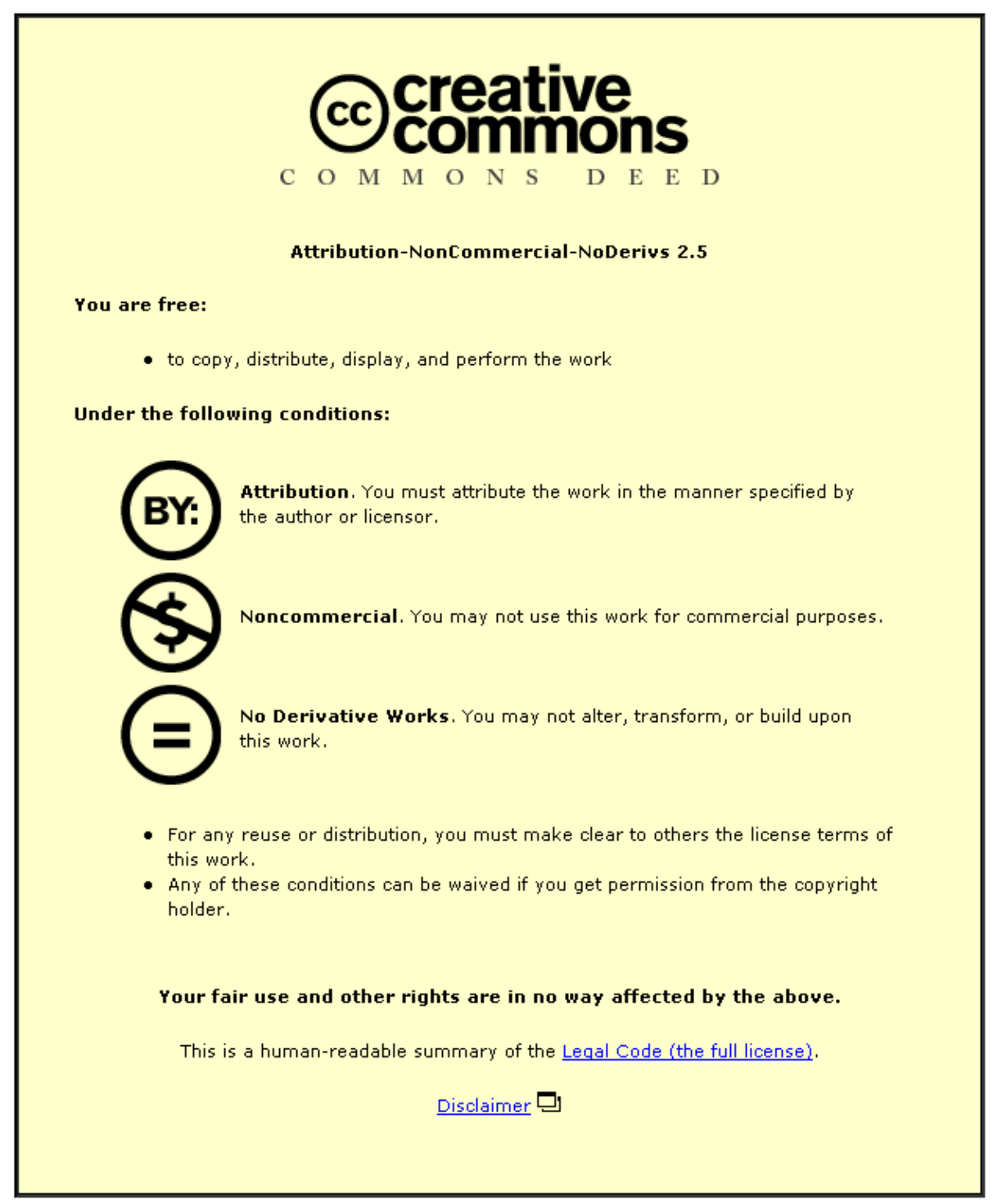

For the full text of this licence, please go to: http://creativecommons.org/licenses/by-nc-nd/2.5/ 


\title{
Computational Modelling of the Anisotropic Conductive Adhesive Assembly Process
}

\author{
David C. Whalley \\ Interconnection Group, Loughborough University, UK (D.C.Whalley@Lboro.ac.uk) \\ Greg Glinsky and Chris Bailey \\ School of Computing and Mathematical Sciences, Greenwich University, UK \\ Johan Liu \\ Division of Electronics Production, Chalmers University of Technology, Sweden
}

\begin{abstract}
Previously developed analytical models of the anisotropic adhesive assembly process have successfully predicted the time for adhesive resin flow out and whether this can be successfully achieved before resin cure. Computational Fluid Dynamics models have also provided significant insights into the effects of the component and substrate bond pad geometry on the resin flow distribution and hence on the resulting final conductive particle distribution. These computational models have however used Newtonian, i.e. non-shear thinning, flow properties for the adhesive materials. This paper will present initial results from the development of more sophisticated models, which include both the non-Newtonian and the temperature dependent flow of the adhesive. Such models can be used to allow a much more detailed investigation of the interactions of the adhesive resin flow characteristics, the component and substrate materials and geometry, and the assembly process parameters. These models, once fully developed and validated, will therefore lead to a better understanding of the assembly process and facilitate establishment of design rules for different applications.
\end{abstract}

\section{Introduction}

Anisotropically conductive adhesives have emerged as an important joining technology in a number of significant application areas, such as flat panel display assembly and smart cards. These materials rely upon the trapping of conductive particles between the conductive pads on the two parts being connected followed by the solidification of the adhesive thereby locking in residual stresses to ensure retention of sufficient contact force to create stable and low resistance electrical connections.

\section{Process steps}

There are a number of key steps identifiable in the ACA assembly process:

Pre-assembly (dispensing or printing of pastes or placement and tack bonding of film materials);

Component placement;

Heating;

Assembly pressure application;

Resin flow and particle compression;

Resin cure;

Assembly pressure release;

Cooling.
This sequence may vary however. For example, for radiation cured resins heating is unnecessary and for thermoplastics there is no cure and cooling must take place before pressure release. The process steps are also not always discrete, there often being significant overlaps, particularly for film materials where the softening due to heating is necessary to allow the resin flow.

In order to design an optimum process for anisotropic adhesive assembly, or to understand the origin of process defects, it is necessary to establish models of the process steps and how they interact to achieve the final geometry and material properties. Williams and Whalley [1] identified many of the important variables in establishing a satisfactory ACA interconnection and Liu [2] summarises the status of scientific understanding of this technology. One of the critical phases in the process is the adhesive flow and how this leads to the conductor particles becoming compressed. This paper will review previously developed analytical adhesive flow models and then present results from Finite Volume (FV) computational models which include both non-Newtonian and temperature dependent flow properties.

\section{Modelling of the adhesive compression process}

The compression/flow of the adhesive resin is divided into two distinct steps [3]. These are: Type I flow, where the bumps on the substrate or components penetrate into the adhesive and the adhesive flows into the gaps between the pads; and Type II flow, where all of the space between the pads has been filled and the adhesive must then flow out from under the component (Figure 1). If Type I flow is not completed then voids will remain under the components, reducing the adhesive bond strength and potentially presenting a significant reliability hazard. Ideally the bond pad sizes and heights and the adhesive thickness should be chosen such that Type II flow just occurs over the whole component area as the conductor particles reach their optimum degree of compression. However, limitations on the choice of these parameters often results in significant Type II flow being necessary. The assembly process must be designed such that this compression is completed before the adhesive resin cures (for a thermosetting adhesive) or re-solidifies (for a thermoplastic), however the process also must not lead to over-compression, and consequent damage, of the conductor particles. 


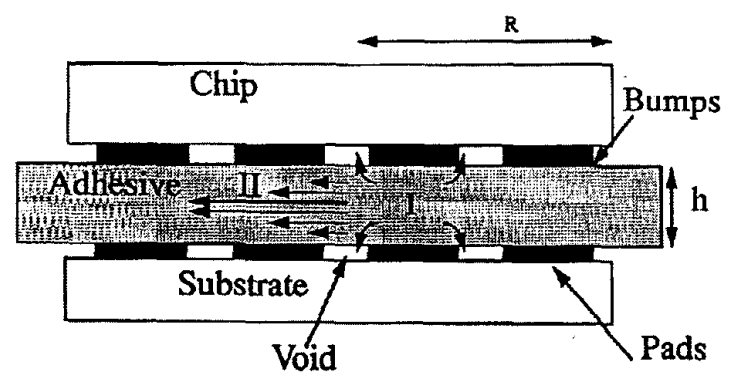

Figure 1. Compression/flow of the adhesive

Ogunjimi et al. [3] approximated the component as being circular, with a purely radial adhesive flow, and used the Scott equation to model the rate of compression of the adhesive, $d h / d t$, during Type II flow. In order to compensate for the fact that the real component is usually square, an effective component radius, $R$, must be calculated such that the area modelled is the same as that of the component, i.e.:

$$
R=\frac{L}{\sqrt{\pi}}=L / 1.772
$$

Equation 1

Where $\mathrm{L}$ is the component length/width.

The flow rate is very dependent on the adhesive properties as well as the applied process assembly force, $F$, and the geometry, i.e.:

$$
\frac{d h}{d t}=-\frac{-n}{2 n+1}\left(\frac{(n+3) F h^{2 n+1}}{2 \pi \eta_{0} R^{n+3}}\right)^{1 / n} \quad \text { Equation 2 }
$$

Where $\eta_{0}$ is the consistency of the adhesive and $n$ is the power law index of viscosity i.e. the viscosity, $\eta$, is given by:

$$
\eta=\eta_{0}(\dot{\gamma})^{n-1}
$$

Equation 3

Equation 2 assumes that $\mathrm{R}>>\mathrm{h}$, however comparison of the prediction of equation 2 with computational fluid dynamics (CFD) models has shown this equation is applicable with only small errors down to $\mathrm{R} / \mathrm{h} \approx 3$ [4]. For $\mathrm{n}=1$, i.e. for a Newtonian fluid, equation 2 simplifies to:

$$
\frac{d h}{d t}=-\frac{2 F h^{3}}{3 \pi \eta_{0} R^{4}}
$$

Equation 4

It is interesting to compare this with the very similar expression which Dudek et al. [5] derived for Type I flow. Dudek et al. also modelled the adhesive compression process analytically, but used a $2 \mathrm{D}$ representation rather than the rotationally symmetric approximation used in [3]:

$$
\frac{d h}{d t}=-\frac{F h^{3}}{\eta b L^{3}}
$$

Equation 5

where $\mathrm{L}$ is the pad/component length and $\mathrm{b}$ the pad width.
The 2D approximation of equation 5 is likely to be more appropriate for assemblies where the pads have a high length to width ratio, such as $\mathrm{LCD}$ to flex connections, but less appropriate for more conventionally shaped IC pads such as those used in smart card applications. Equation 5 however also neglects the effects of shear thinning of the adhesive, which significantly affects the resin flow and it cannot therefore be safely used for Type II flow if the adhesive resin flow is non-Newtonian. Figure 2 shows a comparison of the predicted compressive flow behaviour for these different analytical models for $L=b=5 \mathrm{~mm}, \eta_{0}=100 \mathrm{~Pa} . \mathrm{s}$ and $\mathrm{F}=$ $200 \mathrm{~N}$. Figure 2 clearly shows the high sensitivity of the adhesive flow to even a relatively low degree of shear thinning of the resin (Mannan et. al. [6] reported values for $n$ of 0.4 and 0.9 for two different adhesive film materials). For paste materials this effect is however likely to be somewhat less important as these materials are expected to display approximately Newtonian flow properties.

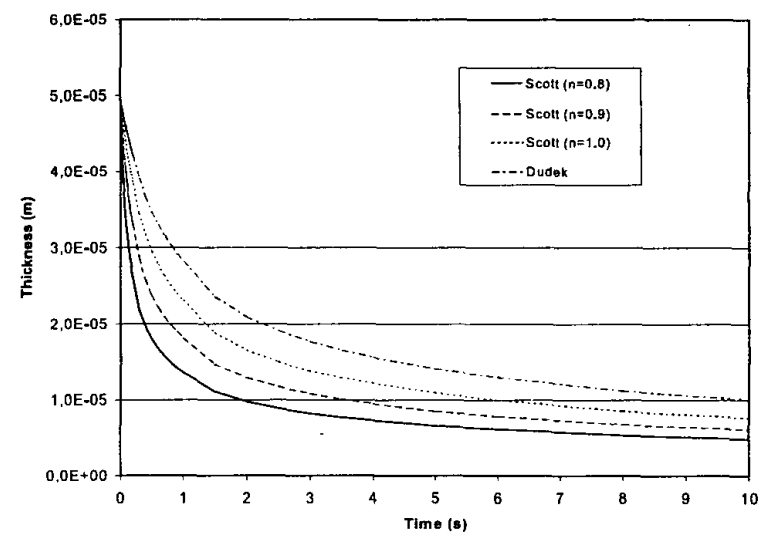

Figure 2. A comparison of analytical models of the compression of an adhesive film

Ogunjimi et. al. [3] also derived the pressure distribution under the component during Type II flow (or across a pad during Type I flow) to be:

$$
P(r)=\frac{n+3}{n+1}\left(R^{n+1}-r^{n+1}\right) \frac{F}{\pi_{0} R^{n+3}} \quad \text { Equation } 6
$$

Where $r$ is the radial distance from the component (or pad) centre. It is interesting to note that neither the viscosity nor the film thickness appear in this equation, so the pressure distribution is expected to remain fairly constant during the assembly process as long as $n$ remains constant. It has been shown by Whalley et al. [7] that the magnitude of this pressure can be sufficient to cause distortion of polymeric substrate materials of a degree comparable with the conductor particle size, potentially resulting in large variations in the degree of particle compression between the centre and edge of a device. This pressure distribution is therefore of great importance where such flexible substrate materials are used.

There have been several other reported studies of modelling of the assembly process. For example, Buratynski [8] modelled the transient heat flows occurring during the curing process and the resulting residual stresses following 
assembly pressure removal and cooling. These models provide some extremely valuable insights, but assume that the adhesive flow has completely finished and that all of the assembly force is therefore carried by the conductor particles before resin curing commences. It has however been shown in [7] that it is likely that this assumption is often invalid, as the metal coated polymer spheres commonly used as conductor particles have insufficient strength to withstand typical assembly forces. Dudek et al. [5], Chiang et al. [9] and Wu et al. [10] have also modelled the residual stresses within the assembly after curing, but have made similar assumptions. Dudek et al. [5] also showed that significant temperature gradients can occur through the adhesive film thickness, which could significantly effect the adhesive flow. This result is supported by the experimental results in [11] where the temperature ramp rate was shown to significantly effect the process. In order to obtain a complete understanding of the ACA assembly process and the effects of the relevant process parameters it is therefore necessary to develop more sophisticated process models. These models should include the detailed geometry, materials properties and also the mechanical interactions between the resin, particles and the parts being interconnected.

\section{Computational Modelling of ACA Resin Flow}

The simulations were performed using PHYSICA, a computational modelling software framework for the solution of multi-physics continuum mechanics problems. The software uses Finite Volume (FV) methods to solve the Navier-Stokes and heat equations. The procedure involves discretising the problem domain geometry into a grid of finite cells with nodal points located at the centres, at which flow and heat variables e.g. velocity, pressure and temperature are stored.

All of the models presented are for a $3 \mathrm{~mm}$ square chip and an initial adhesive film thickness of $70 \mu \mathrm{m}$. The simplicity of the domain geometry allows an orthogonal structured grid of brick shaped elements to be used. The adhesive is modelled as a cuboid and two orthogonal symmetry planes through the central axes of the chip are used to reduce the model size by a factor of four without reducing accuracy, thereby saving memory storage and computational effort. The two adjacent model edges representing the cut symmetry planes are modelled by constraining the gradients of all property variables, together with the fluid and heat flux in the normal direction to the boundary, to zero. The two opposite boundaries represent the chip edges at which the adhesive outflow occurs. Here the boundary pressure is constrained to a constant reference value of zero. This ensures a continuous outflow of adhesive at the two chip edges. The upper and lower horizontal plane surfaces represent the chip and substrate surfaces respectively. Here prescribed velocities of half that of the initial adhesive film compression rate are applied in opposite directions at both the chip surface and the substrate surface.

The flow model implemented allows simulation of nonNewtonian behaviour with both shear rate and temperature dependent viscosity. The variable viscosity is approximated by a piece-wise linear variation in the values, which are stored at the nodal locations at the cell centres. The temperature dependency relationship, constitutive model and the calulation of the shear rate are implemented by means of a user written Fortran subroutine that is integrated with the PHYSICA code. The shear rate $\dot{\gamma}$, is calculated using the velocity gradients that are automatically evaluated and stored by the PHYSICA code after each iteration of the solution procedure. These gradients are based on the solution of the nodal velocity field.

Figure 3 shows a cross sectional representation of the model and the boundary conditions applied to it. The required assembly force to achieve the specified compression velocity is determined from the FV modelling results by integrating the calculated pressures over the adhesive area.

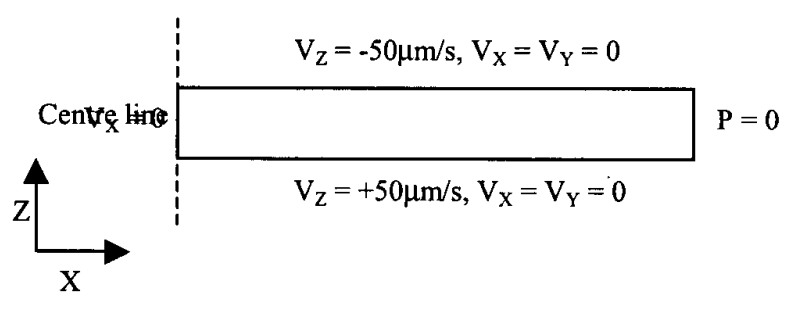

Figure 3. Boundary conditions for the FV model

\section{Uniform temperature models}

In order to validate the FV model an initial comparison was made with results from the previously used analytical model. For the Newtonian flow model a constant viscosity of $100 \mathrm{~Pa}$.s was used and for the shear thinning model values of $\eta_{0}=294$ and $\mathrm{n}=0.625$ were used. The initial adhesive compression rate specified was $100 \mu \mathrm{m} / \mathrm{s}$.

Figure 4 shows a comparison of the predicted pressure distributions from equation 6 and from the FV model of the process. It can be seen that there is close agreement between the two types of model for both Newtonian and nonNewtonian flow. The required assembly force predicted by the FV models to achieve this compression rate was $0.98 \mathrm{~N}$ for the Newtonian flow case and $0.66 \mathrm{~N}$ for the shear thinning case. Figure 5 shows the predicted pressure distribution for the Newtonian FV model (the chip centre is at the top left).

Figure 6 shows a comparison of the through film thickness flow velocity profile at the chip edge for the Newtonian and non-Newtonian FV models. Although the shear thinning of the adhesive results in a lower assembly force for a given initial compression rate, it can be seen in Fig. 6 that the flow velocity distributions are quite similar for the two cases.

\section{The effect of a temperature gradient on flow}

As noted earlier, significant through film temperature gradients may occur in certain assembly processes. A complete model of the process must therefore include this effect as the adhesive viscosity is highly temperature dependent and a temperature gradient through the adhesive film thickness was expected to create an asymmetrical flow distribution. This may have positive or negative effects on the 


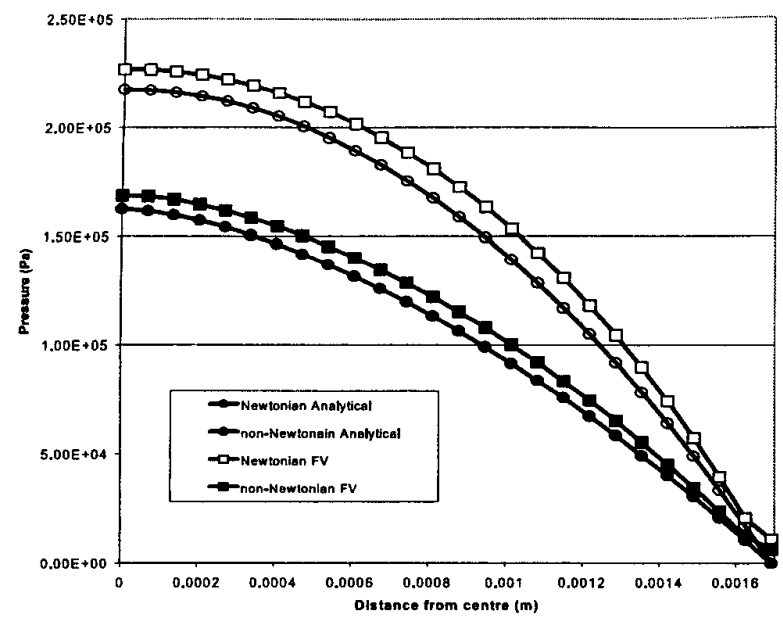

Figure 4. Comparison of predicted pressure distributions under a flip-chip during adhesive flow

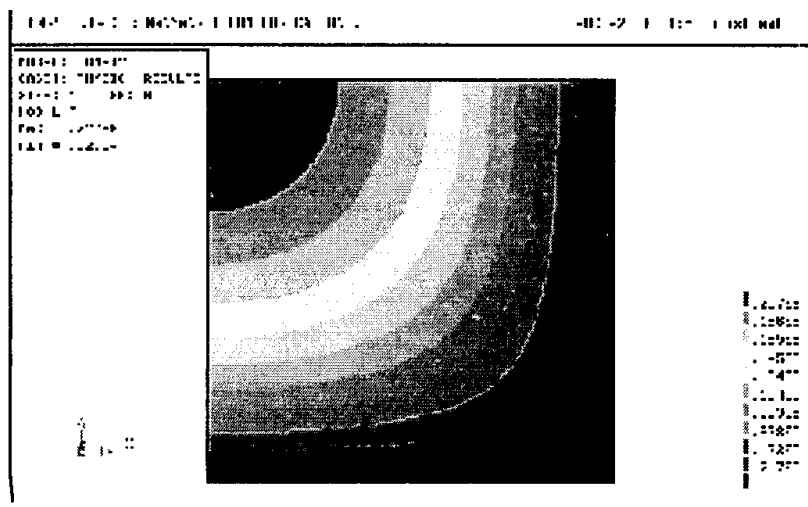

Figure 5. Predicted pressure distribution for Newtonian adhesive flow at constant temperature

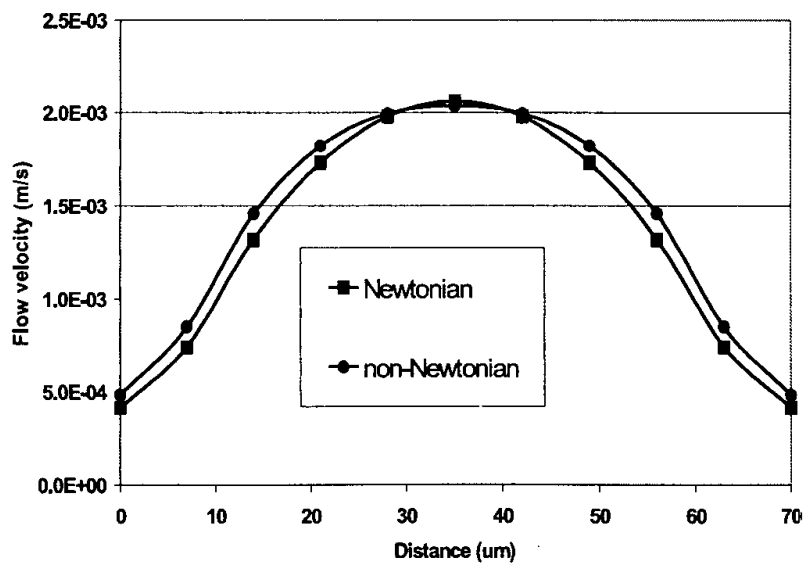

Figure 6. Comparison of predicted through thickness flow velocity at chip edge final particle distribution depending on their initial distribution and the relative direction of the temperature gradient. Temperature gradients of $20^{\circ} \mathrm{C}$ or $60^{\circ} \mathrm{C}$ were therefore added to the models to evaluate their effects. This was achieved by adding applying fixed temperature boundary conditions of $\pm 10^{\circ} \mathrm{C}$ and $\pm 30^{\circ} \mathrm{C}$ relative to the reference temperature to the upper and lower surface of the adhesive. The temperature dependence of viscosity presented in [6] was used i.e.:

$$
\eta=\eta_{0} e^{-0.093 \Delta T}
$$

Equation 7

PHYSICA is capable of simultaneously solving for both temperatures and flow making this addition to the model quite straightforward. The model was first run using a Newtonian flow characteristic and as expected the pressure distribution was not affected significantly. The required force to achieve the set compression rate however rose slightly, i.e. to $1.01 \mathrm{~N}$ for the $20^{\circ} \mathrm{C}$ gradient and $1.02 \mathrm{~N}$ for the $60^{\circ} \mathrm{C}$ gradient. This small increase in assembly force, despite the large drop in viscosity at the hotter side of the adhesive film, is thought to be due to the flow being constrained in a thinner layer. This effectively reduces the value of $h$ in equation 2 , which it can be seen will rapidly increase the required force for a given compression rate. As was also expected the flow velocity profile became asymmetrical between the top and bottom of the chip. Figure 7 shows how the horizontal flow velocity at the edge of the chip varies through the film thickness and Figure 8 shows plots of the flow magnitude distributions from the chip centre to the edge. The $Z$ axis in Figure 8 has been enlarged by a factor of about 5 in relation to the $X$ axis in order to make the flow distribution easier to see.

This analysis was then repeated for the same temperature dependence of viscosity on temperature but also with a nonNewtonian flow model. Figure 9 shows the predicted flow velocity profiles at the chip edge and it can be seen that the combined effect of shear thinning and a temperature gradient is much higher than for a temperature gradient alone. For the $20^{\circ} \mathrm{C}$ temperature gradient the predicted assembly force rose to $0.70 \mathrm{~N}$, but for the $60^{\circ} \mathrm{C}$ gradient it had fallen again to $0.56 \mathrm{~N}$, presumably because the reduction in viscosity due to the combined effects of increased temperature and shear thinning were sufficient to exceed the effect of the flow being constrained into a much thinner layer.

\section{Conclusions}

It has been shown that the pressure distributions predicted using the Newtonian and non-Newtonian FV models both agree closely with those from the previously developed analytical flow model. This gives confidence in the FV modelling approach and therefore in the results predicted for more complex situations which the analytical model cannot address. These results show that it is important to include nonNewtonian flow behaviour in models and provide further evidence that a more sophisticated modelling approach is required in order to allow a full understanding of the effects of the assembly process on the final properties of the assembly. As the adhesive flow and the mechanical distortion of the substrate, component and conductor particles are closely 
coupled, "multi-physics" models, which simultaneously combine the time varying temperature distribution within the assembly with non-Newtonian fluid flow and the solid mechanics interactions are therefore necessary. These are now in the process of being developed.

\section{Acknowledgements}

David Whalley gratefully acknowledges the financial support of this work provided by the Royal Academy of Engineering through the Engineering Foresight Awards Scheme.

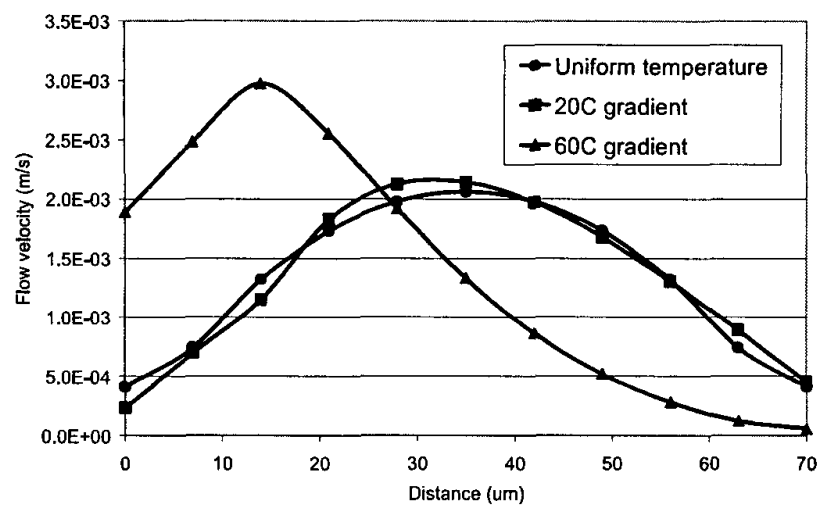

Figure 7. The effect of a temperature gradient on the through thickness flow velocity for Newtonian now

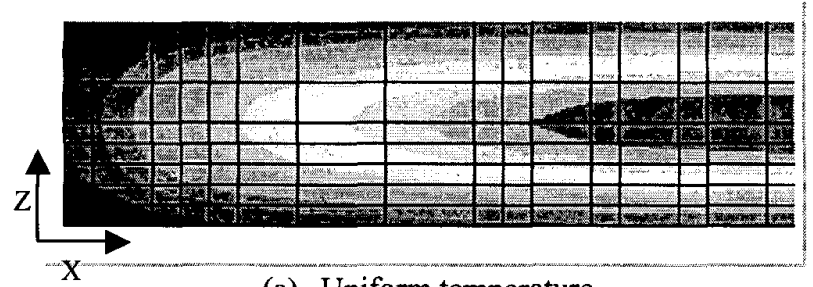

(a) Uniform temperature
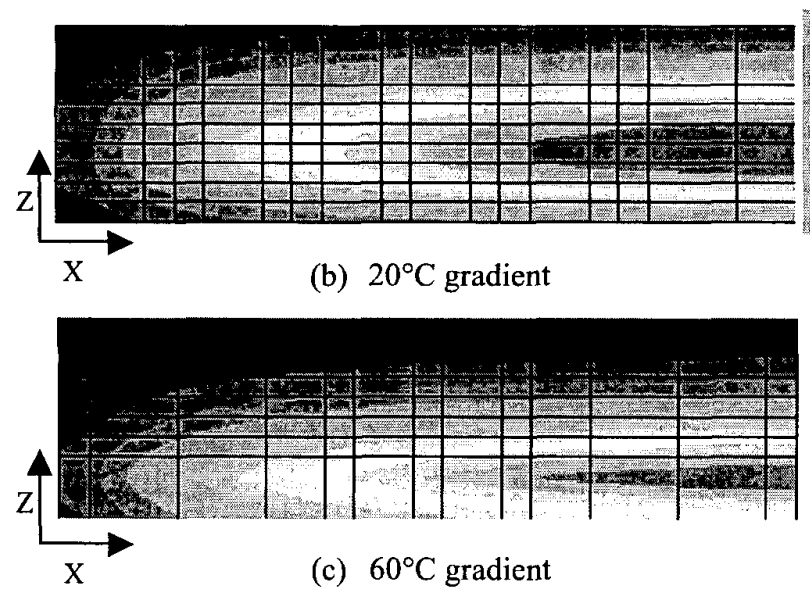

Figure 8. The effect of a temperature gradient on the flow magnitudes for Newtonian flow

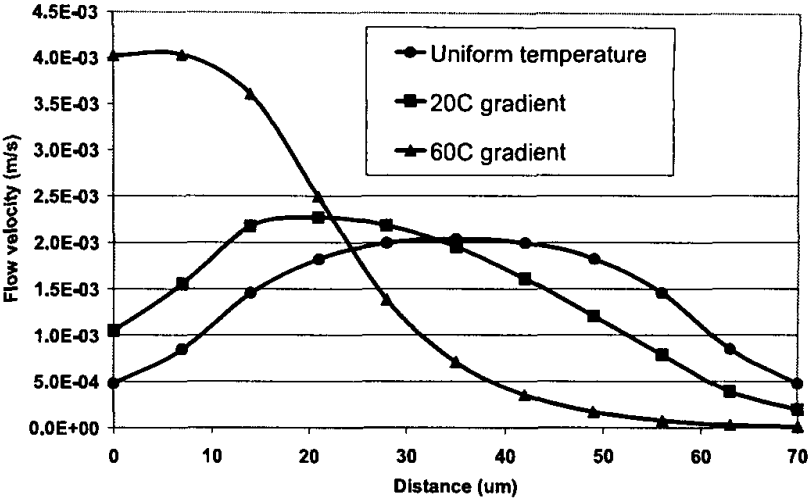

Figure 9. The effect of a temperature gradient on the through thickness flow velocity for non-Newtonian flow

\section{References}

1. Williams D.J. and Whalley, D.C., "The effects of conducting particle distribution on the behaviour of anisotropic conducting adhesives - non uniform conductivity and shorting between connections" Journal of Electronic Manufacturing, Vol. 3 (1993), pp. 85-94

2. Liu, J., "ACA bonding technology for low cost electronics packaging applications - current status and remaining challenges" Soldering and Surface Mount Technology, Vol. 13, No. 3 (2001), pp. 39-57

3. Ogunjimi, A. O., Mannan, S. H., Whalley, D. C. and Williams, D. J., "The assembly process for anisotropic conductive joints - some new experimental and theoretical results", Journal of Electronic Manufacturing, Vol. 5 (1995), pp. 263-272

4. Mannan, S.H., Whalley, D.C., Ogunjimi, A.O., and Williams, D. J., "Assembly of planar array components using anisotropic conducting adhesives - a benchmark study: Part II - Theory" IEEE Transactions on Components, Packaging and Manufacturing Technology Part C, Vol. 19 (1996), pp. 264-269

5. Dudek, R., Meinel, S., Schubert, A., Michel, B., Dorfmüller, L., Knoll, P.M. and Baumbach, J., 'Flow characterization and thermo-mechanical response of anisotropic conductive films" "IEEE Transactions on Components and Packaging Technology, Vol. 22 (1999), 177-185

6. Mannan, S.H., Williams, D.J. and Whalley, D.C., "Some Optimum Anisotropic Conductive Adhesive Properties for Flip Chip Interconnection", Journal of Materials Science: Materials in Electronics, Vol. 8 (1997), pp. 223231

7. D. C. Whalley, L. Chen and J. Liu "Modelling of the Anisotropic Adhesive Assembly Process" Proceedings of the Fourth International Symposium on Electronic Packaging Technology, Beijing, August 2001, pp. 319324, ISBN 0-7803-9811-4 
8. Buratynski, E.K., "Thermomechanical modelling of direct chip interconnection assembly", Journal of Electronic Packaging Vol. 115 (1993), pp. 382-391

9. Chiang, K.N., Chang, C.W. and Lin, J.D., "Analysis of ACA/ACF Package Using Equivalent Spring Method" IEEE Electronics Packaging Technology Conference, Singapore, 2000, pp. 110-116

10. Wu, C.M.L., Liu, J. and Yeung, N.H., "The effects of bump height on the reliability of ACF in flip-chip" Soldering and Surface Mount Technology, Vol. 13 (2001), pp. $25-30$

11. Gustafsson, K., Mannan, S.H., Lai, Z., Liu, J., Whalley, D.C. \& Williams, D.J., "The Effect Of Temperature Ramp Rate On Flip-chip Joint Quality And Reliability Using Anisotropic Conductive Adhesive On FR-4 Substrate" Proceedings of the 47th Electronic Components and Technology Conference, San Jose, 1997, pp. 561-566 